\title{
LE RÉGIME DU RHÔNE DANS L'ANTIQUITÉ ET AU HaUt Moyen ÂGE
}

\author{
Mireille Provansal, Jean-François Berger, Jean-Paul Bravard, Pierre-Gil Salvador, \\ Gilles Arnaud-Fassetta, Hélène Bruneton, Anne Vérot-Bourrély
}

\begin{abstract}
Mots-clés. Morphogenèse du lit fluvial, crues, variations hydrologiques, histoire du climat, géoarchéologie fluviale.
Key-words. River bed morphogenesis, floods, hydrologic variations, climate history, river geoarchaeology.

Résumé. Les mutations de la plaine alluviale du Rhône du lac Léman à la mer, entre le Ve s. avant J.-C. et le Haut Moyen Âge, sont analysées par le biais des données sédimentaires, pédologiques et géomorphologiques de nombreux sites archéologiques urbains et ruraux. L'étude séparée du secteur amont, médian et aval souligne la cohérence du fonctionnement fluvial au cours de cette période et met en évidence l'importance du facteur climatique. Deux crises majeures affectent le fonctionnement et les lits du fleuve, au cours du Premier Âge du Fer et du Haut Moyen Âge. Elles sont séparées par une longue période de calme hydrologique relatif entre le II s. avant J.-C. et les $V^{e}$ $V$ s. après J.-C. Une recrudescence des crues affecte néanmoins le Rhône supérieur et inférieur entre le Ier s. avant J.-C. et le Ir s. après J.-C. Sur le Rhône moyen, cette dégradation est décalée entre le début du Pror s. et le début du III s. après J.-C. L'impact de ces fluctuations sur les sites archéologiques est discuté.
\end{abstract}

\begin{abstract}
The variations of the Rhône alluvial plain, from lake Leman to the Mediterranean sea, between the $5^{\text {th }}$ century BC and the Early Middle Ages, are analysed through pedology, sedimentology and geomorphology on several urban and rural archaeological sites. Separated studies of the upper, middle and lower parts of the valley reveal the consisiency of the river behaviour and the importance of climatic factor during this period. Two major crisis affect the hydrologic system and the river beds during the Iron Age I and the Early Middle Ages. A relatively long hydrologic pause remained between the $2^{\text {nd }}$ century and the $4^{\text {th }} 5^{\text {th }}$ century $A D$. However increasing floods happened in the upper and lower Rhône valley during the $1^{\text {st }}$ century $B C$. and the $1^{\text {st }}$ century $A D$. On the middle Rhône, this crisis is delayed between the beginnings of the $1^{\text {st }}$ and $3^{\text {rd }}$ century $A D$. The impact of these variations on the archaeological sites is examined.
\end{abstract}

L'étude des mutations des milieux fluviaux rhodaniens et de leurs relations avec les sociétés riveraines a été initiée il y a une quinzaine d'années sur le HautRhône français. Le développement des recherches paléoenvironnementales sur le Rhône moyen et inférieur, sur site et hors site, permet aujourd'hui de proposer un bilan de l'histoire "naturelle » du fleuve. Sans a priori déterministe, il peut aider à une réflexion sur les contraintes inhérentes à l'occupation des plaines allu- viales et sur les réponses qu'y ont apportées les sociétés antiques.

\section{LES CARACTÈRES GÉOGRAPHIQUES DU BASSIN-VERSANT RHODANIEN}

Du lac Léman à la mer, le fleuve parcourt environ $450 \mathrm{~km}$, dans des contextes morphologiques et hydrologiques différents (fig. 1). 


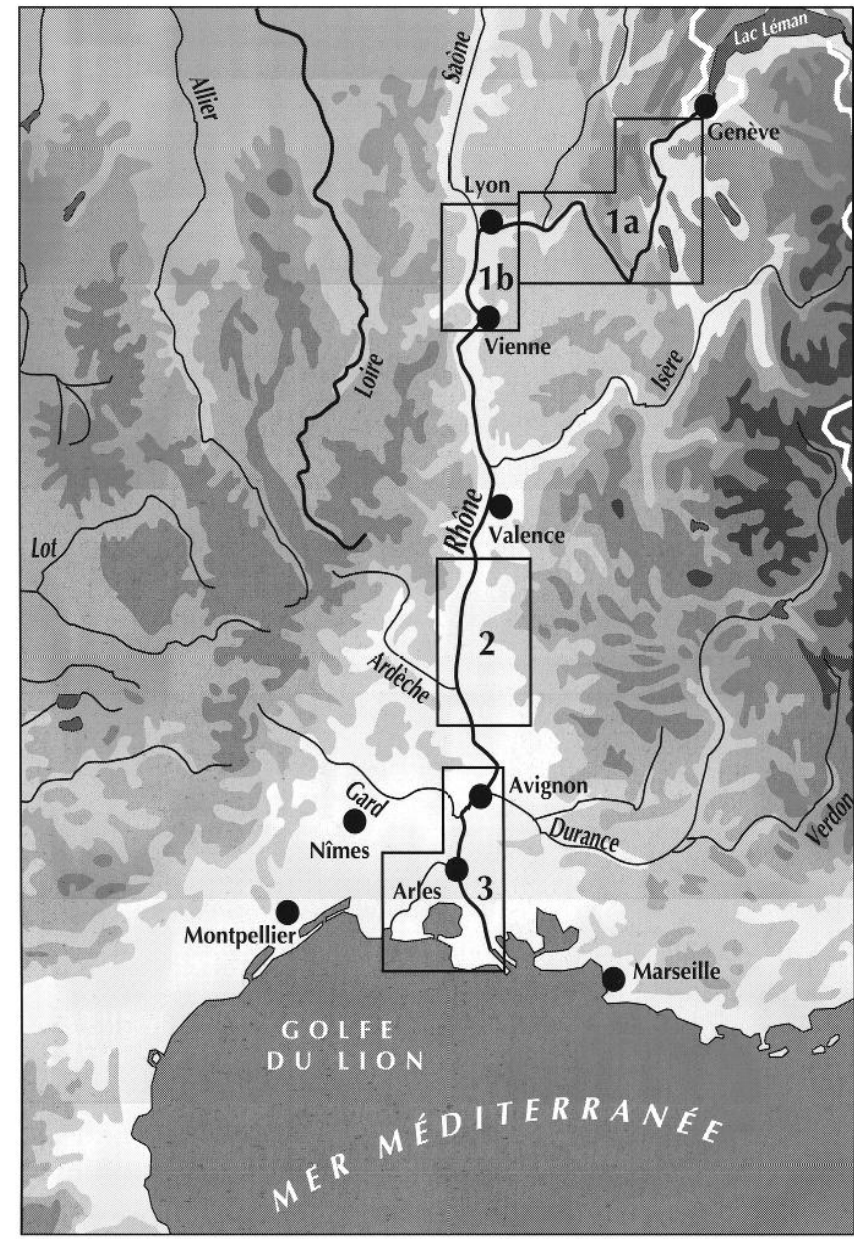

Fig. 1 - Le Rhône, du lac Léman à la mer, contexte orographique el localisation des secteurs étudiés : 1, le Haut-Rhône français (1a, le Rhône du Léman au Bugey-Isle Crémieux; 1b, le Rhône en LyonnaisViennois) ; 2, le Rhône moyen de Valence à Orange ; 3, le Bas-Rhône d'Avignon à la mer.

\section{LE HAUT-RHÔNE FRANÇAIS : UN FLEUVE SOUS L'INFLUENCE DES ALPES}

De la cuvette lémanique au confluent de l'Ain, le Haut-Rhône est fortement conditionné par l'héritage de la dernière glaciation quaternaire : le fleuve coule dans une succession d'ombilics de surcreusement glaciaire et de gorges ou verrous résistants. À l'aval de cette confluence, il reçoit un important apport sédimentaire grossier qu'il étale dans une plaine alluviale large et bien calibrée jusqu'en aval de Lyon. À partir de Givors, le Rhône s'engage de nouveau dans une succession de défilés et de bassins, mais l'ancienneté du façonnement de cette partie de la vallée fait que le fleuve moderne n'est pas contraint par son cadre (fig. 1 et 2).

Le régime hydrologique du Haut-Rhône est d'abord nivo-pluvial, puis pluvial grâce aux apports jurassiens et surtout à l'arrivée de la Saône à Lyon. La proximité de hauts reliefs donne des crues-éclairs, en particulier lorsque des pluies importantes coincident avec la fonte des neiges en fin d'hiver et au printemps; mais les cuvettes lacustres et les vastes plaines de remblaiement jouent un rôle majeur dans l'écrêtement des crues.

Si la tendance à long terme est à la régularisation du profil en long hérité de la phase de déglaciation, cette évolution n'a pas été linéaire au cours de l'Holocène. Très proche des sources sédimentaires et disposant d'une forte énergie, le Rhône a en effet enregistré une série de fluctuations hydroclimatiques qui ont affecté son bilan sédimentaire.

\section{LE RHÔNE MOYEN, DE VALENCE À AVIGNON}

La pente longitudinale assez forte, de l'ordre de 0,7 à 0,8 pour 1000 , engendre des vitesses d'écoulement importantes. De plus, le franchissement des seuils rocheux et des défilés de Tain-Tournon, Cruas, Donzère et Mornas-Mondragon raidit localement le profil en long. En aval de ces obstacles, les lits fluviaux s'élargissent, parfois sur plusieurs kilomètres. Ils sont alors favorables aux accumulations sédimentaires et aux mutations rapides des milieux fluviaux (fig. 3).

Les affluents préalpins de rive gauche (Drôme, Roubion/Jabron, Berre, Lez, Aygues et Ouvèze) fournissent des sédiments abondants, qui renouvellent les apports sédimentaires grossiers. De vastes cônes de déjection ont progressivement rejeté le cours du fleuve aux pieds des versants ardéchois. Les seuls affluents importants de rive droite sont l'Ardèche et la Cèze.

Le régime subit l'influence des affluents oroméditerranéens de rive gauche, sujets à des crues d'automne ou de printemps dont les débits extrêmes et la compétence sont élevés. La mise en charge généralisée de ces bassinsversants est génératrice de crues centennales à très vaste champ d'inondation, susceptibles de remodeler l'ensemble des lits fluviaux. 


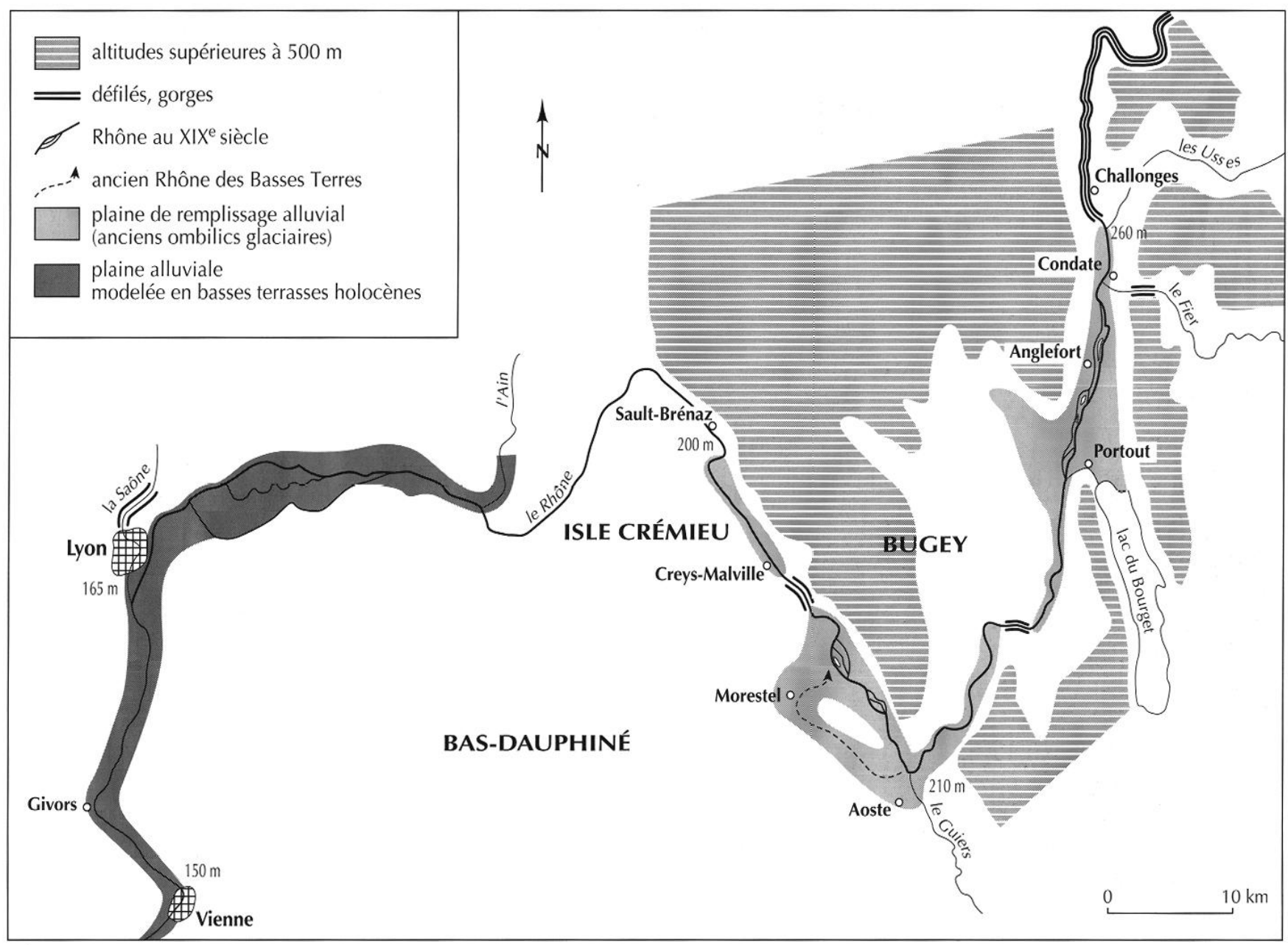

Fig. 2 - Le Haut-Rhône : hydrographie, contexte géomorphologique et localisation des principaux sites.

\section{LE RHÔNE INFÉRIEUR, D'AVIGNON À LA MER}

La vallée du Rhône inférieur reste caractérisée par la traversée d'éperons résistants qui raidissent localement le profil en long, entre les bassins d'Orange, d'Avignon et d'Arles (fig. 4). La réduction de la pente du fleuve à l'approche du delta (3 m NGF à Arles) favorise l'accumulation et la persistance de marécages dans le lit majeur. Le nombre et la position des bras dans le delta ont fluctué à plusieurs reprises depuis le Néolithique; les bras de Saint-Ferréol et d'Ulmet, aujourd'hui atterris, évacuaient l'essentiel des eaux du fleuve à l'époque romaine. L'alluvionnement fait prograder ${ }^{2}$ un rivage dont la

2. La progradation est le déplacement vers l'aval des dépôts sédimentaires dans un lit fluvial. À l'aval d'un delta ou en milieu littoral, ce processus entraîne une avancée du trait de côte. géographie reste néanmoins mal connue au début de notre ère.

L'élévation du niveau marin, amplifiée par les déformations négatives du sol, est encore perceptible au cours du Moyen Âge : le niveau marin relatif antique est donc encore situé quelques décimètres sous le niveau actuel, autorisant un meilleur drainage des sols.

Subissant les influences complémentaires de l'ensemble du bassin-versant rhodanien, le régime fluvial actuel ne connaît pas de véritables étiages : le fleuve dispose donc d'une capacité de transport et d'une puissance nette élevées. Les deux derniers affluents, la Durance et le Gardon, sont sujets à de fortes crues d'automne et de printemps qui fournissent des sédiments abondants et grossiers. La mise en charge généralisée du bassin-versant est génératrice de crues centennales à très vaste champ d'inondation susceptibles de remodeler ou de déplacer le lit fluvial. 


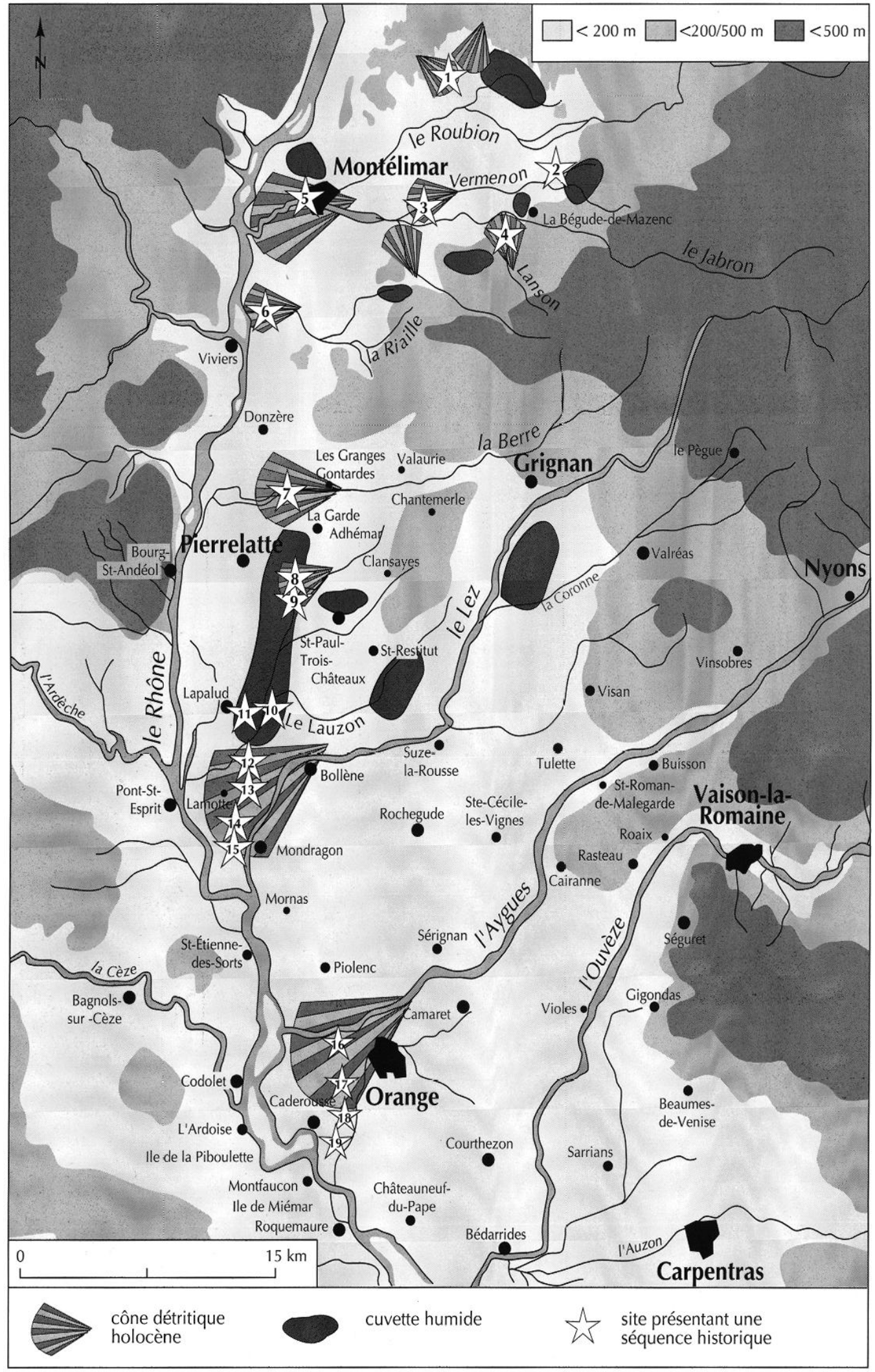

Fig. 3 - Le Rhône moyen : hydrographie, contexte géomorphologique et localisation des principaux sites. 


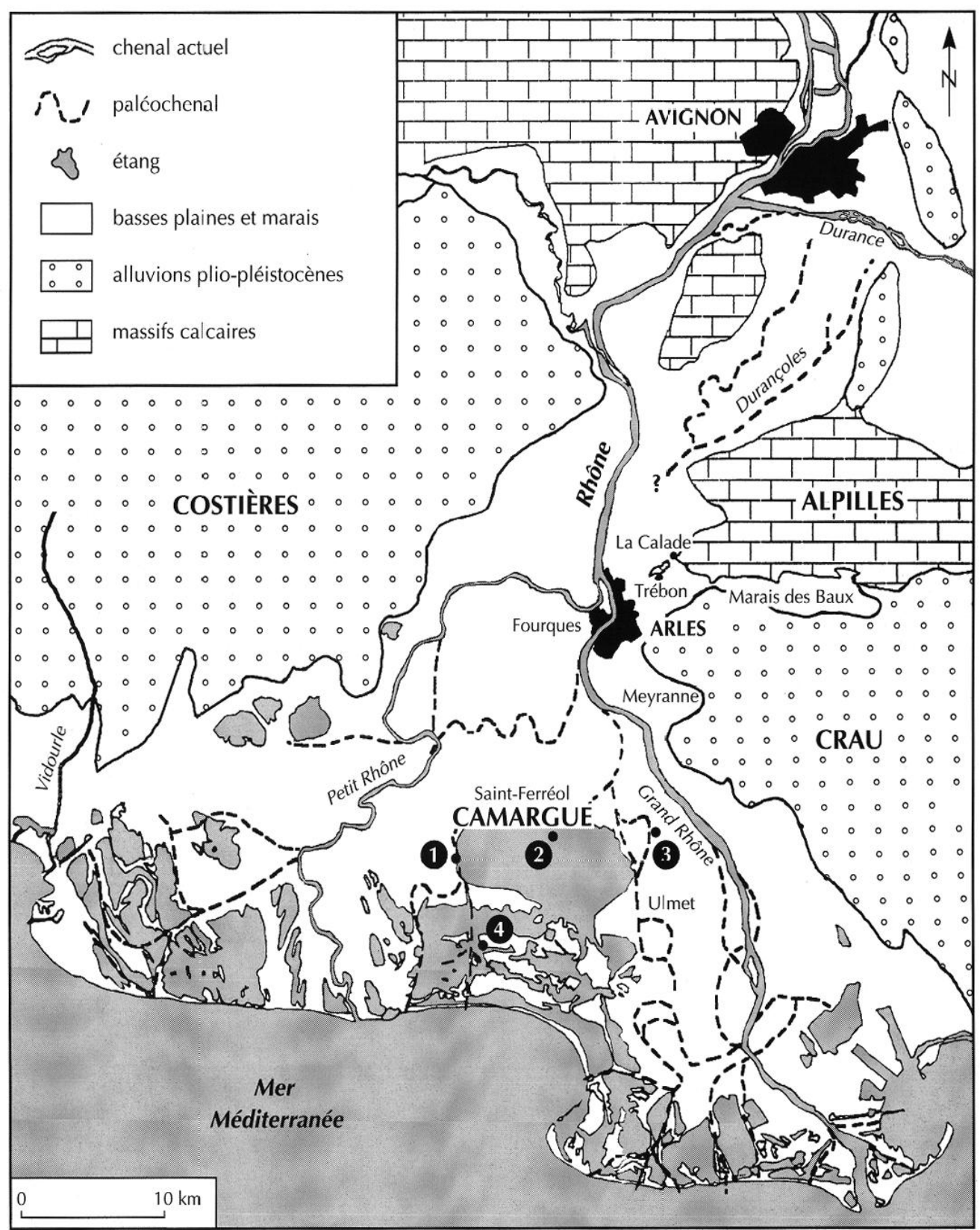

Fig. 4 - Le Bas-Rhône : hydrographie, contexte géomorphologique et localisation des principaux sites : 1, Le Carrelet ; 2, Cabassole ; 3, La Capelière ; 4, Mornès.

\section{MÉTHODOLOGIE}

Les traces du fonctionnement fluvial de la période romaine et du Haut Moyen Âge sont dispersées et partielles. Leur signification peut être ambiguë (un dépôt de crue élevé est-il significatif d'une crue exceptionnelle? d'un obstacle local ? d'un exhaussement tendanciel du lit ?) et nécessite d'être confrontée à d'autres critères d'interprétation. Il importe donc de combiner les indicateurs archéologiques, morphosédimentaires et pédologiques qui peuvent permettre de faire la part de l'épisodique, généralement bien accepté par les sociétés riveraines, et du long terme, qui pourrait entraîner des adaptations significatives de l'occupation des sols. 


\section{LES CRITÈRES MORPHOSÉDIMENTAIRES}

Ils sont de deux types, fournissant des informations différentes dont l'exploitation en termes paléohydrologiques peut être ambiguë lorsqu'ils sont étudiés séparément.

- Les dépôts alluviaux sont dus aux inondations. Leur altitude, liée au niveau atteint par l'eau, peut traduire l'abondance hydrologique de l'événement (crues exceptionnelles) ou l'encombrement du chenal principal (îles, embâcles ralentissant l'écoulement). La grossièreté et l'épaisseur des dépôts sont proportionnelles à l'énergie du flux liquide et à l'éloignement de la source sédimentaire. Mais elles peuvent dépendre aussi de l'importance de l'érosion des sols dans le bassin-versant...

- La géométrie et le nombre des lits fluviaux sont affectés de changements pluridécennaux à séculaires (« métamorphoses fluviales »), qui font alterner deux types de paysages (Bravard, Petit, 1997) :

. un chenal profond et sinueux (" style à méandres "), des berges peu élevées, un champ d'inondation réduit et peu marécageux ; cette situation correspond à un régime régulier et à des débits liquides abondants et peu chargés ;

- un chenal large et peu profond, à bras multiples, et encombré de bancs sableux ( "style tressé »), des berges élevées, un champ d'inondation étendu et marécageux, une tendance à la défluviation; cette situation correspond à un régime irrégulier, caractérisé par des crues hautes et fréquentes (retour de 1 à 2 ans), avec forte charge solide grossière.

Le temps de réponse du style fluvial aux effets des variations climatiques est encore mal connu et dépend de la taille, de la pente et de l'agencement du bassinversant.

\section{LES CRITÈRES PÉDOLOGIQUES}

Les épisodes de stabilité fluviale sont marqués par le développement de sols plus ou moins évolués. La comparaison avec les conditions climatiques et anthropiques actuelles permet de reconstituer les conditions paléoenvironnementales de leur développement (Courty et al., 1989 ; Courty, 1994; Berger, 1996a) : pluviométrie, évapotranspiration, bon drainage naturel ou engorgement des sols, mise en culture...
Le type de pédogenèse dans la plaine d'inondation est dépendant du niveau de la nappe phréatique alluviale : les sols hydromorphes sont souvent associés à la remontée du niveau moyen des nappes et à un style fluvial en tresse ; à l'inverse, les sols bien drainés sont associés à un enfoncement du chenal fluvial. Mais l'abaissement artificiel des nappes peut être obtenu par un réseau de fossés de drainage comme ce fut le cas pendant la période romaine. Enfin, la topographie de la plaine et la nature du substrat peuvent aussi favoriser la persistance de milieux humides.

L'étude des cortèges malacologiques (Magnin, in : Berger, 1996a, p. 113-130) apporte des renseignements fiables sur les conditions d'humidité locales du milieu et sur la structure de la végétation au niveau stationnel (taux et type de couverture végétale).

\section{LES CRITÈRES ARCHÉOLOGIQUES}

La localisation et les transformations des sites archéologiques mettent en évidence les variations tendancielles du milieu fluvial relevant de variations séculaires du climat (métamorphoses des lits, bilan hydrique des sols), plutôt que les épisodes liés à des phénomènes météorologiques brefs (crues).

Les crues ne sont en effet une contrainte majeure que si la pente du lit leur confère une vitesse génératrice de risque pour les structures bâties et les hommes; elles sont donc moins redoutables dans la plaine aval, dont la pente longitudinale est inférieure à 0,1 pour 1000 . La submersion temporaire des champs ne perturbe pas l'activité agricole et apporte des limons fertilisants.

La « métamorphose » vers un «style tressé » est plus contraignante, car elle est le corollaire de mutations qui affectent l'ensemble du système fluvial pendant plusieurs générations : en engorgeant les sols de façon durable, elle oblige à des travaux de drainage ou d'entretien réguliers des réseaux et, éventuellement, à relever le plancher des structures (habitats, canalisations, voiries).

La combinaison des différents indicateurs énumérés ci-dessus aboutit à définir, schématiquement, trois situations-types :

- les périodes de "pause hydrologique », caractérisées par une sédimentation très faible à nulle, sont en relation avec des crues peu fréquentes et un chenal bien incisé ; 
les sols alluviaux sont bien développés, avec des caractéristiques dépendant de la durée de la période et de la nature du contexte climatique et anthropique ;

- les périodes de " crise hydrologique », caractérisées par une sédimentation rapide, un exhaussement important du plancher fluvial, le développement du tressage dans le lit mineur et des marécages dans la plaine d'inondation, sont associées à des crues fréquentes et à un haut niveau moyen de la nappe ; l'érosion latérale est aggravée sur les berges, en marge de la bande de tressage en voie d'exhaussement. Les processus pédologiques sont ralentis ou stoppés ;

- les périodes de " transition ", caractérisées par une sédimentation lente, permettent le développement d'un sol alluvial peu évolué, mais épais. Le fleuve est soumis à de fortes crues épisodiques mais conserve un chenal bien incisé.

\section{LE RHÔNE, SECTORISATION GÉOMORPHOLOGIQUE}

La confrontation des données obtenues à l'échelle régionale, du lac Léman à la mer, permet de proposer une histoire paléoenvironnementale du Rhône antique. La diversité des contextes géomorphologiques et climatiques, et l'inachèvement des recherches expliquent la persistance de divergences entre les différents segments : les connaissances sont en effet tributaires de situations conjoncturelles, qu'il s'agisse de grands travaux ou de fouilles réalisées en milieu urbain. On présentera donc séparément des tronçons au comportement différent : de Genève à Lyon, le fleuve a une évolution spécifique, influencée par les héritages glaciaires; de Lyon à la mer, l'histoire du fleuve, plus homogène, autorise néanmoins une présentation séparée en trois secteurs (Lyon-Vienne, Valence-Avignon, Avignon-Méditerranée).

\section{UN COMPORTEMENT HÉTÉROGÈNE ENTRE LE LÉMAN ET L'ENTRÉE DANS LES PLAINES DE L'EST LYONNAIS}

Le Rhône a un tracé relativement jeune puisqu'il pourrait s'être établi depuis la fin de la période glaciaire, qui connut une avancée des glaciers hors des Alpes. Ce trait explique le caractère inachevé de la morphologie du fond de vallée et la grande variabilité amont-aval du comportement fluvial en tre Genève et le confluent de l'Ain. Jusqu'à la sortie du Jura, l'évolution de la vallée est dominée par le remblaiement des cuvettes glaciaires; elle devient plus complexe à partir du secteur Bugey-Isle Crémieu.

\section{Du LÉman aU Bas-DAUPHINÉ}

Le fleuve manifeste une tendance continue au remblaiement des cuvettes lacustres qui occupaient les ombilics glaciaires depuis environ 15000 ans. Le remblaiement alluvial est caillouteux, alimenté par une abondante charge de fond provenant des affluents torrentiels (Arve, Usses, Fier, Guiers, Valserine), ou organominéral de type « arrière-marais fluvial ».

L'accumulation ennoie les vestiges archéologiques gallo-romains : les carrières de Challonges, après leur abandon au milieu du I ${ }^{\text {er }}$ s. après J.-C. (Savay-Guerraz, 1985), la ville de Condate, fossilisée sous plusieurs mètres de sédiments, des stèles à l'emplacement du barrage d'Anglefort en Chautagne (Dufournet, Chevallier, 1981) et l'atelier de potier de Portout, le long du canal de Savières qui assure la communication entre le lac du Bourget et le Rhône. La remontée des niveaux lacustres, mise en parallèle avec l'aggradation ${ }^{3} \mathrm{du}$ lit du Rhône, atteindrait environ $1 \mathrm{~m}$ par millénaire, sans être pour autant continue (Magny, Richard, 1985).

Dans la plaine de Morestel-Le Bouchage, dernier grand ombilic glaciaire, le remblaiement est entaillé par un train de méandres, bordé d'installations galloromaines (Bravard, 1986). Ces méandres fossiles permettent d'évaluer les conditions de la navigation antique, qui furent sans doute plus aisées dans un chenal unique, à faible pente et sinueux, que dans le cours d'eau " à tresses " moderne et contemporain (Bravard, 1996). Après une très probable défluviation du Rhône, survenue aux environs des $\mathrm{II}^{\mathrm{e}}$-IV $\mathrm{IV}^{\mathrm{e}} \mathrm{s}$. après J.-C., ces formes sont fossilisées sous une tourbière à partir des VI ${ }^{\mathrm{e}}$-VIII ${ }^{\mathrm{e}} \mathrm{s}$. après J.-C. (Roberts et al., 1997). Le Rhône adopte alors un tracé plus court et se fixe au pied du Jura méridional. L'absence de tout site antique sur son tracé actuel suggère qu'il est très probablement postérieur à l'époque gallo-romaine (R. Royet, comm. orale).

3. Accumulation verticale des couches sédimentaires aboutissant à une élévation du sommet des dépôts. 


\section{Le SEGTEUR BugeY-Isle CrémIEU}

Le petit bassin de Creys-Malville, limité par des versants calcaires résistants, est essentiellement alimenté par les cônes de déjection de la Brivaz et de la Perna, les apports en provenance de l'amont étant toujours restés bloqués dans la cuvette des Basses Terres du BasDauphiné. Cette configuration conduit à distinguer deux types d'évolution (Salvador, 1991 ; Salvador et al., 1993). - À l'amont, le tracé du chenal est stable depuis le Néolithique moyen au moins et l'accumulation est dominante. Mais on identifie deux types de fonctionnement fluvial :

. au Premier Âge du Fer, et de façon plus hypothétique, au début de l'occupation romaine, entre 40 et 30 avant J.-C., le fleuve est caractérisé par une activité réduite : faible taux de sédimentation, limitée aux zones basses, et dépôts de basse énergie ;

- de courts épisodes de péjoration hydrologique caractérisent le Second Âge du Fer et la fin du I ${ }^{\text {er }}$ s. avant J.-C. : réactivations de chenaux, sédimentation plus importante et plus grossière ( 1 à $3,6 \mathrm{~mm} / \mathrm{an}$ ), extension spatiale des surfaces alluvionnées traduisant sur les sites concernés des crues plus puissantes; une péjoration plus longue débordant sur la fin du $\mathrm{I}^{\mathrm{er}} \mathrm{s}$. et le $\mathrm{II}^{\mathrm{c}} \mathrm{s}$. après J.-C. peut être envisagée, dans la mesure où les sites de plaine sont abandonnés entre 70 et 150 après J.-C. alors qu'ils se maintiennent sur les marges du bassin, mais aucune preuve déterminante n'a pu être avancée; l'habitat regagne la plaine de 150 après J.-C. à la fin de l'époque gallo-romaine, alors que les modalités de l'alluvionnement demeurent comparables.

- À l'aval des cônes de déjection, la présence de plusieurs paléoméandres est révélatrice de la mobilité latérale du fleuve. La dernière phase d'activité, la seule enregistrée, voit migrer le fleuve de la bordure de la plaine (rive droite) vers son axe actuel, depuis une époque antérieure au Premier Âge du Fer.

\section{LE RHÔNE EN LYONNAIS-VIENNOIS, DU CONFLUENT DE L'AIN À VIENNE}

Le confluent de l'Ain renouvelle les apports sédimentaires grossiers favorisant les manifestations de métamorphoses fluviales sur le tronçon de l'Ain à Lyon. Dans le secteur de Vienne, le lit majeur du Rhône est, au contraire, relativement étroit puisqu'il est à peine deux fois plus large que le fleuve actuel. Il est probable que les variations des flux hydriques et minéraux y ont produit des réponses morphologiques marquées sur le plan vertical, faute d'une possibilité de dissipation latérale de l'énergie. L'ampleur des fluctuations fluviales y est donc plus nette qu'à Lyon (environ $3 \mathrm{~m}$ pendant la période historique) et l'ampleur des aménagements humains y est considérable.

Les deux sites permettent une périodisation précise de l'histoire du fleuve (Bravard et al., 1990, 1997) (fig. 5).

Au cours du Premier Âge du Fer jusqu'au début de La Tène, le plancher caillouteux rhodanien s'exhausse (Arlaud et al., 1994) et, à Lyon, repousse le lit de la Saône au pied de la colline de Fourvière car l'affluent dispose d'une puissance inférieure (Macé et al., 1991).

À La Tène et jusqu'au règne d'Auguste, vers $-10 /+10$, une phase de pause hydrologique induit une incision $\mathrm{du}$ lit fluvial, permettant l'occupation de la plaine d'inondation. À Lyon, le Rhône se déplace vers l'est, libérant le site de la presqu'île qui reste néanmoins inondable; la Saône ourre un nouveau chenal (approximativement sur son tracé actuel) et libère l'actuelle île Saint-Jean en rive droite au début du $\mathrm{I}^{\text {er }}$ s. après J.-C. (Bravard et al., 1997). À Vienne, le chenal du Rhône, vraisemblablement unique, est incisé d'environ $3 \mathrm{~m}$ car le lit majeur ne porte pas de sédiments fins issus de débordements. Ces données sont cohérentes avec les cotes des égoûts de SaintRomain-en-Gal.

À partir de 30-15 avant J.-C., une hydrologie plus vigoureuse induit des inondations plus fréquentes. Des alluvions fines, datées de la fin du $\mathrm{I}^{\mathrm{er}} \mathrm{s}$. avant au $\mathrm{I}^{\mathrm{er}} \mathrm{s}$. après J.-C., témoignent de séquences de débordement alternant avec des épisodes de balayage de crues. À Lyon, les dépôts de crues les plus anciens sont fossilisés entre deux niveaux de fréquentation augustéens $(7$ à $30 \mathrm{~cm})$, puis au cours du règne de Tibère ( 6 à $15 \mathrm{~mm} / \mathrm{an}$ ); les derniers dépôts alluviaux sont fins et précèdent une installation datée du règne de Claude $(4 \mathrm{~cm} / \mathrm{an})$. À Vienne, cette phase se manifeste également par des érosions de berges et la construction de cônes de déjection en contrebas des ruisseaux de Saint-Gervais (Vienne) et de Servandière (Saint-Romain-en-Gal). 
Fig. 5 - La Saône et le Rhône au début de notre ère à Lugdunum.

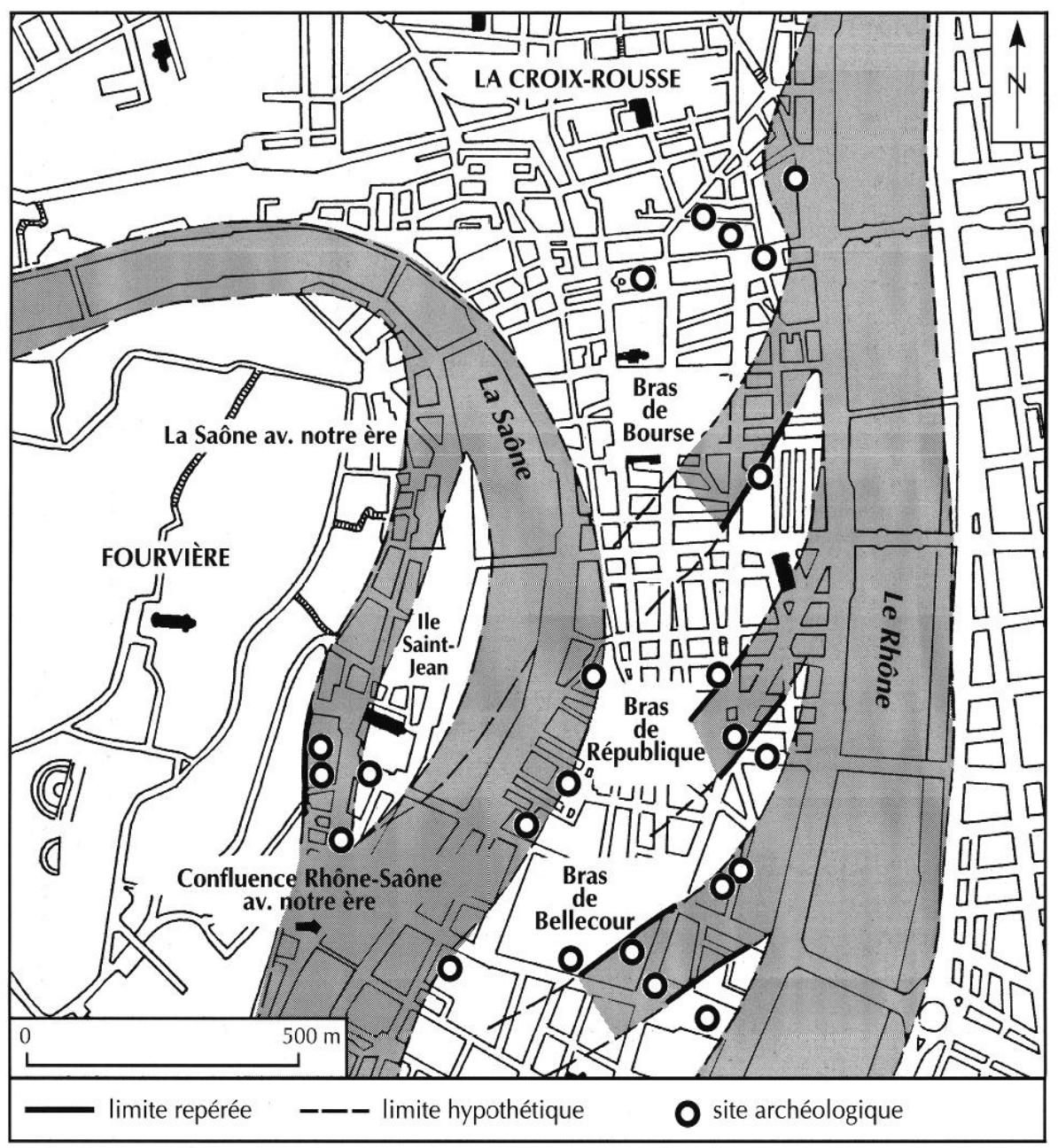

À la même période (en relation avec la recrudescence des crues ?), des réaménagements urbains sont caractérisés par des remblaiements. Sous le règne de Claude, l'ensemble des sites lyonnais de la presqu'île est exhaussé à l'aide d'un remblai caillouteux d'une épaisseur d'environ $50 \mathrm{~cm}$, qui permet d'étendre l'urbanisation et la met à l'abri des inondations (Arlaud et al., à paraître). Au pied de Fourvière, les Lyonnais s'efforcent de rattacher l'île Saint-Jean, nouvellement formée : dépôts de crues et remblais anthropiques se succèdent de la seconde moitié du I ${ }^{\mathrm{er}} \mathrm{s}$. au milieu du IIII $\mathrm{e}$ s. après J.-C. (Arlaud et al., 1994). À Vienne, la ville et les entrepôts sont reconstruits après 40 après J.-C. : $2,80 \mathrm{~m}$ plus haut que les niveaux augustéens à Vienne et de 1,50-2 m à Saint-Romain-en-Gal.

De la fin du IV s. après J.-C. au début du Haut Moyen Âge, les inondations du Rhône et de la Saône reprennent. Alors que les sites de la rive droite de la Saône enregis- trent une occupation continue de l'époque galloromaine à nos jours, les autres gisements de la presqu'île présentent un hiatus archéologique. Celui-ci n'est toujours pas expliqué et une troncature artificielle des dépôts n'est pas exclue.

\section{LE RHÔNE MOYEN, DE VALENCE À AVIGNON}

Il existe encore peu d'analyses effectuées directement dans le lit du fleuve dans cette partie du cours, à l'exception de la plaine d'Orange qui a bénéficié des prospections réalisées lors de la construction du TGVMéditerranée. L'information provient essentiellement des lits fluviaux préalpins et de leurs cônes détritiques à la confluence avec le Rhône (fig. 3). Elle concerne donc les apports solides et liquides en provenance des Préalpes calcaires du sud. Des études pluridisciplinaires réalisées 
dans l'arrière-pays, en position de pied de versant (milieux colluviaux), permettent de compléter l'image des paysages dans l'Antiquité et le Haut Moyen Âge (état des sols et taux de recouvrement végétal notamment), d'évaluer le rôle des agrosystèmes sur l'érosion et de proposer des relations entre les zones amont et aval des bassins-versants tributaires du Rhône (Berger, 1996a).

Le Rhône conditionne l'évolution du style fluvial et des basses plaines alluviales de ses affluents préalpins, dont il constitue le niveau de base ; ainsi, lorsqu'il s'enfonce sous le niveau de son lit majeur actuel (période d'incision linéaire), il induit, par érosion régressive, un ajustement du profil en long de l'ensemble de ses affluents.

L'analyse des réseaux de fossés antiques des très basses plaines fluviales apporte une information complémentaire sur la mise en valeur de ces milieux et surtout sur l'évolution des aquifères au cours des deux derniers millénaires, qui peut être mise en parallèle avec les études morphosédimentaires et paléopédologiques (Berger, Jung, 1996).

Globalement, la période considérée semble caractérisée par une activité hydrologique et sédimentaire irrégulière, dans laquelle se distinguent des périodes de dérèglement hydrosédimentaire très marquées, des périodes de transition ou d'instabilité hydrologique et des périodes de pause hydrosédimentaire. Le Premier Âge du Fer, la période comprise entre la fin du $\mathrm{I}^{\mathrm{er}}$ et le III $^{e}$ s. après J.-C. et la transition Antiquité tardive/Haut Moyen Âge sont caractérisés par une crise hydrologique majeure susceptible de modifier profondément les milieux fluviaux.

Entre le VIT et le $V^{e}$ s. avant J.-C., les lits fluviaux s'exhaussent de plusieurs décimètres à $1 \mathrm{~m}$ en moyenne. Les taux de sédimentation apparaissent très élevés dans les lits majeurs au sud du Tricastin et dans la plaine d'Orange (3-4 mm/an). Des sols peu évolués, très hydromorphes, se succèdent dans les plaines fluviales. Le style fluvial « en tresse » sur l'ensemble des affluents préalpins du Rhône (Riaille, Bramefaim, Vermenon...) témoigne de la progradation de la charge de fond et de débits solides et liquides abondants.

Entre le $V^{e}$ et le III s. avant J.-C., une période de transition ou d'instabilité hydrologique lui succède. Avant le début du IVes. avant J.-C., l'alluvionnement se ralentit de manière importante par rapport au Premier Âge du Fer. Les processus pédologiques, avec un début de brunification ${ }^{4}$, semblent se développer dans les lits majeurs (sites de Bollène-le Pont de la Pierre, CrestBourbousson). De nombreuses installations humaines s'implantent alors à proximité des lits fluviaux et ne semblent pas subir de crues trop fréquentes. L'instabilité fluviale est momentanément réactivée entre le milieu du IV ${ }^{c}$ et la fin du III ${ }^{\mathrm{e}} \mathrm{s}$. (ou le début du II ${ }^{\mathrm{c}} \mathrm{s}$. avant J.-C.) sur le Lez et entre les niveaux d'occupation de La Tène ancienne ( $V^{\mathrm{e}}$-début $I V^{\mathrm{c}} \mathrm{s}$. avant J-C.) et ceux de La Tène récente ou du début de la période romaine dans la plaine du Tricastin. Ces signatures montrent la persistance de crues épisodiques.

De la fin III'-début II' s. avant J.-C. au début du ${ }^{\text {er }}$ s. après J.-C., les milieux fluviaux enregistrent une pause hydrosédimentaire bien marquée. Elle se caractérise par une nette réduction de l'alluvionnement, favorable à l'expression des processus biologiques et pédologiques, et par une incision linéaire marquée. La formation d'horizons calciques ${ }^{5}$ bien exprimés est à mettre en relation avec une augmentation des processus d'évaporation et d'évapotranspiration et une ambiance plus sèche décrite par l'analyse des cortèges malacologiques. Cette période est associée à une intense colonisation des basses plaines alluviales de la moyenne vallée du Rhône (réorganisation de l'habitat rural), car une vingtaine d'aménagements ruraux (enclos notamment) sont identifiés sous les alluvions ultérieures, souvent à proximité immédiate des lits mineurs : sites protohistoriques de Montélimar, du Logis-de-Berre, de Surel, des Brassières (Berger, 1996a).

De la fin du $I^{e r}$ s. après J.-C. à la fin du III ${ }^{e}$ s. après J.-C., une nouvelle détérioration hydrologique se caractérise par un fort exhaussement des lits fluviaux ( 50 à $80 \mathrm{~cm}$ ), des taux de sédimentation très élevés dans le sud du Tricastin et la plaine d'Orange (3-4 mm/an) (fig. 6) et

4. Processus de pédogenèse de type climatique caractéristique des régions à climat atlantique tempéré ou semi-continental. Il produit des humus de type mull, et un horizon $B$ ou Bt très pauvre en matière organique et coloré en brun par les oxydes de fer.

5. Horizon d'accumulation de carbonate de calcium $\left(\mathrm{Ca} \mathrm{Co}_{3}\right)$. Cette accumulation peut ĉtre due à la remontéc du carbonate compris dans les nappes aquifères sous forme dissoute au cours d'une phase d'évaporation importante. 


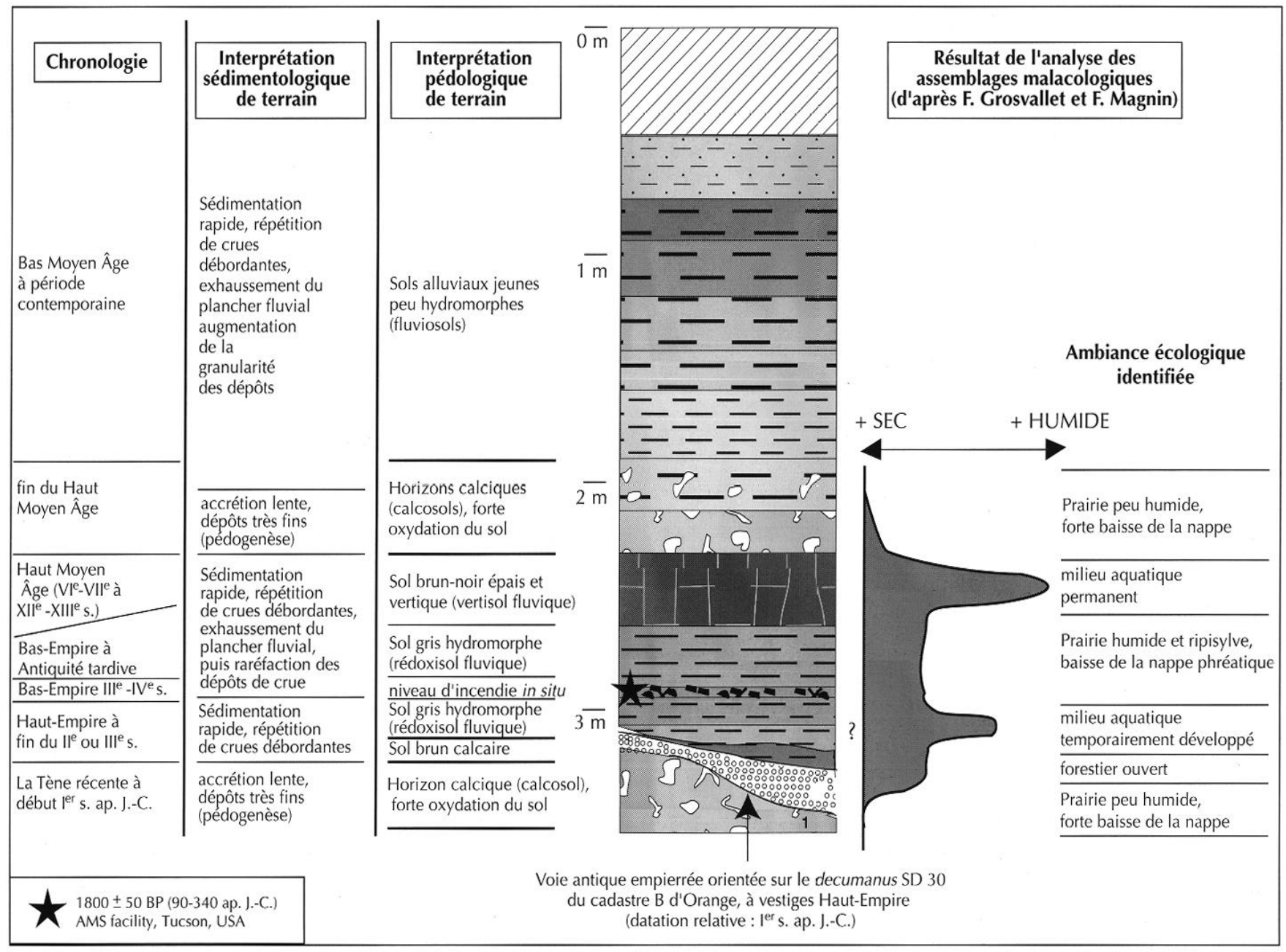

Fig. 6 - Corrélation entre les données pédosédimentaires (J.F. Berger) et les données malacologiques (F. Magnin), sondage de Caderousse-les Crémades (confluence Rhône-Aygues, Vaucluse).

des signatures torrentielles aux pieds des versants préalpins. Des sols peu évolués et très hydromorphes se développent dans les plaines alluviales, témoignant de la remontée des nappes (Berger, 1996b). Leurs cortèges malacologiques confirment les modifications du bilan hydrique et montrent un pic d'humidité, indicateur d'une remontée durable du niveau des aquifères entre la seconde partie du $\mathrm{I}^{\text {er }}$ s. et le III $^{\mathrm{e}} \mathrm{s}$. dans la plaine d'Orange. Une partie des réseaux de drainage et de voirie paraissent au moins momentanément abandonnés dans les basses plaines et fossilisés sous les alluvionnements répétés.

Entre la fin du III et la fin $d u V^{*}$ s. après J.-C., le ralentissement de l'alluvionnement (même s'il peut encore être important localement comme à la confluence Rhône-Lez) correspond à une nouvelle phase de transition. Les processus pédologiques semblent se développer jusque dans les lits mineurs, dans lesquels s'observe un début de brunification. Ils traduisent une amélioration du drainage (forte oxydation et ouverture de la porosité, formation d'horizons calciques peu développés) qui pourrait refléter une reprise de l'incision linéaire et l'augmentation des processus d'évapotranspiration, donc de la température moyenne (Berger, à paraître). De courtes phases d'alluvionnement, bien calées au cours des $I^{c}$ et $V^{c} s$. après J.-C. dans le sud du Tricastin et la plaine d'Orange, viennent perturber localement le fonctionnement des agrosystèmes (multiplication des curages, scellement de voiries et de fossés sous quelques 

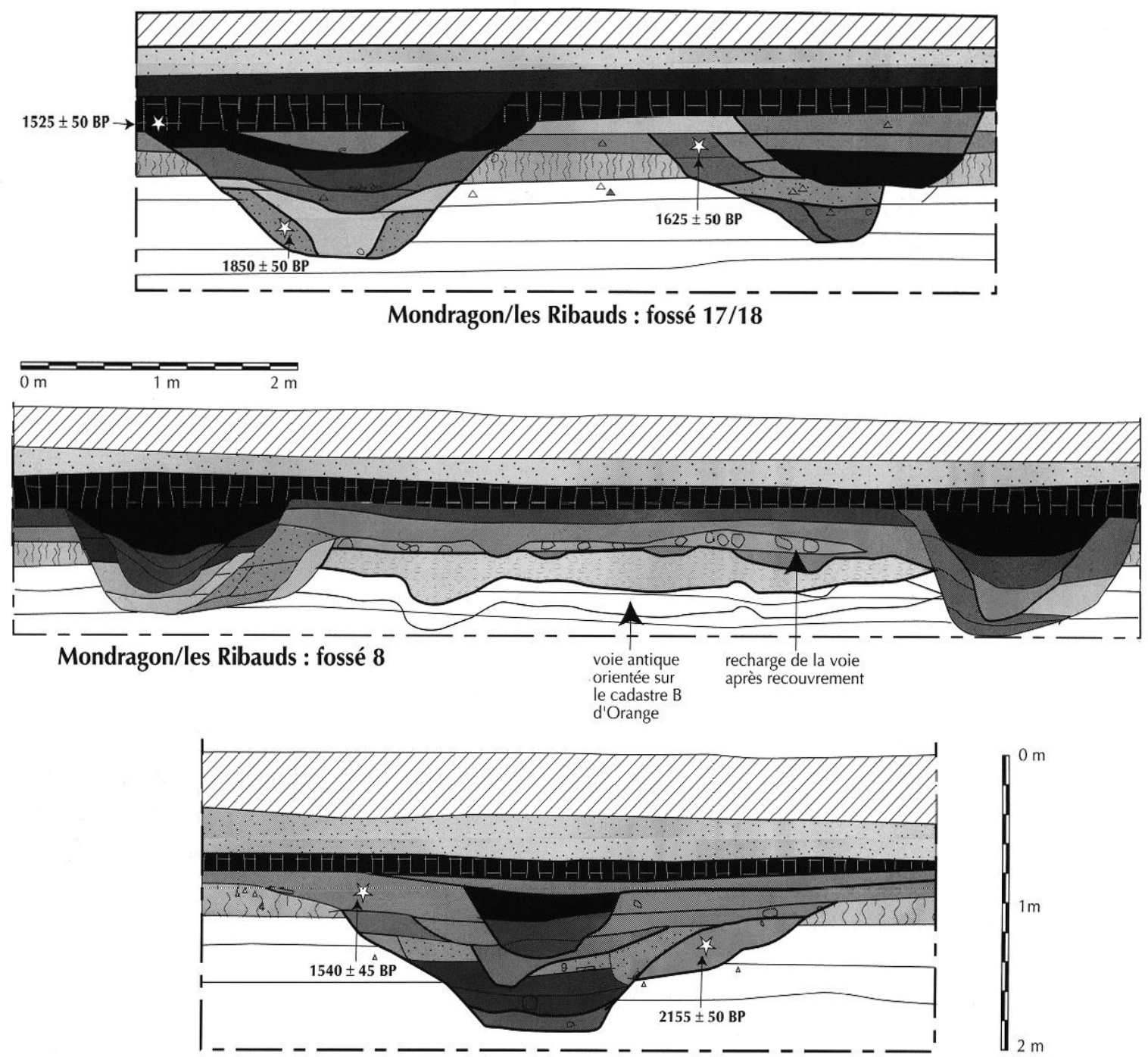

Mondragon/les Brassières : fossé 66

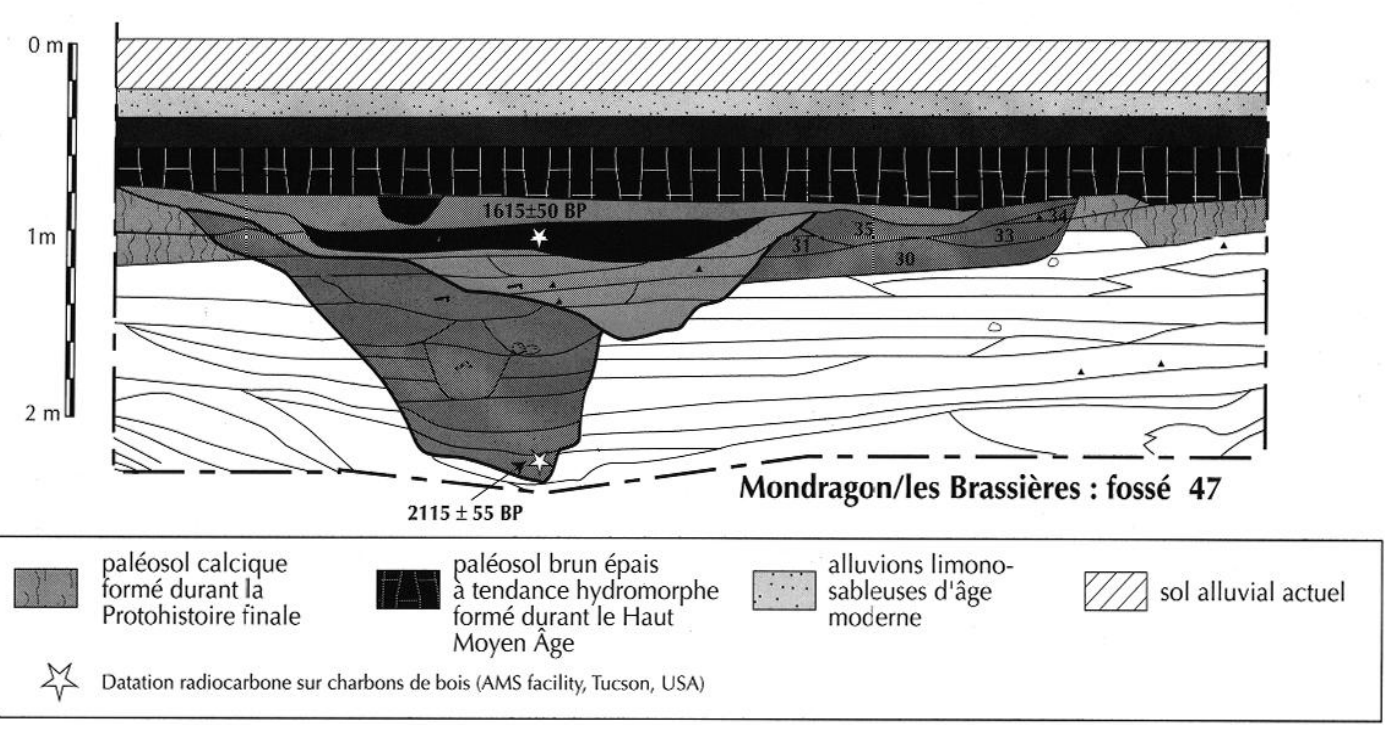

Fig. 7 - Enfouissement des fossés de drainage antiques de la plaine alluviale du Tricastin sous les alluvions de l'Antiquité tardive et du début du Moyen Âge (modifiée d'après J.F. Berger et C. Jung). 
centimètres d'alluvions) et préfigurent la crise hydroclimatique majeure de l'Antiquité tardive et du début du Moyen Âge (fig. 7).

De la fin du $V^{e}$-début $V I^{e}$ s. au $V I I^{e}-V I I^{e}$ s. après J.-C., un fort exhaussement des lits fluviaux s'observe à nouveau $(40-50 \mathrm{~cm})$. Les taux de sédimentation sont alors très élevés dans le sud du Tricastin et la plaine d'Orange (3-4 mm/an). L'instabilité des affluents préalpins est très forte. L'ensemble du réseau de drainage de la moyenne vallée du Rhône est abandonné dans la seconde partie du VIe $\mathrm{s}$. ou la première moitié du VII ${ }^{\mathrm{e}} \mathrm{s}$. ; il est rapidement scellé par quelques décimètres d'alluvions (fig. 7).

\section{LE RHÔNE INFÉRIEUR, D'AVIGNON À LA MER}

Les fouilles de sauvetage à Avignon et Arles, les prospections liées aux aménagements linéaires (dont le TGV) et le programme archéologique "Delta du Rhône " ont permis d'acquérir une information géomorphologique et sédimentaire sur la plaine alluviale du Rhône et sur le chenal fluvial lui-même, qui a été traversé par un carotlage profond danss le delia. Mais l'épaisseur de la sédimentation ne permet pas, généralement, de remonter antérieurement au Second Âge du Fer.

\section{L'ÉVOLUTION DU CHENAL}

La confrontation des sources écrites antiques avec les observations paléoenvironnementales permet plusieurs remarques au sujet de l'évolution du chenal au cours de la période étudiée.

$\mathrm{Au} \mathrm{III}^{\mathrm{e}} \mathrm{s}$ avant J.-C., un passage de Polybe ( $c f$. Leveau, supra, p. 100-101) indique que le Rhône est encombré d'îles dans un secteur dont le point amont se trouve à "quatre jours de marche » de son embouchure. Hannibal, que la présence de l'armée romaine empêche de passer le Rhône trop près du delta, a dû remonter jusqu'à Roquemaure (?) " pour traverser le fleuve en un endroit où le chenal est unique ". L'interprétation de la présence d'îles est complexe : il peut s'agir des manifestations d'un tressage fluvial, significatif d'un déséquilibre hydrosédimentaire. Mais, dans un lit à pente faible chargé en sédiments fins, la multiplication des chenaux peut relever d'une " anastomose fluviale " dans un contexte de stabilité hydrosédimentaire. Ce phénomène a pu être amplifié localement par les défilés rocheux ou l'arrivée d'affluents qui ralentissent l'écoulement dans les plaines d'Orange, d'Avignon et de BeaucaireVallabrègues. L'utilisation du texte de Polybe mérite donc vraisemblablement d'être confortée par la sédimentologie des dépôts de cette période pour qu'une interprétation paléohydrologique soit possible.

La variation du nombre des bras et des embouchures du fleuve est un autre élément à verser à ce "dossier ». Les bras de Saint-Ferréol et d'Ulmet, aujourd'hui atterris, évacuaient l'essentiel des eaux du fleuve au cours de l'Âge du Fer (Lhomer et al., 1981). Un troisième bras a pu momentanément fonctionner au $\mathrm{I}^{\text {er }} \mathrm{s}$. avant J.-C. : l'iconographie et un texte de Strabon semblent indiquer une instabilité des embouchures à la fin du II ${ }^{e} s$ avant J.-C., significative de défluviations, donc d'un déséquilibre hydrosédimentaire (cf. Vella et al., infra, p. 137-138). Le chenal de Saint-Ferréol a été traversé dans le delta par les carottages du Carrelet (fig. 8). Au cours des deux premiers siècles de notre ère, le lit du Rhône y apparaît fortement incisé $(12 \mathrm{~m})$, avec une charge de fond peu grossière et peu abondante. Son remblaiement s'amorce lentement au cours de la seconde moitié du II ${ }^{\mathrm{e}}$ s., puis très rapidement à partir du $V^{e}$ s. après J.-C. (Arnaud-Fassetta, 1998).

\section{L'ÉVOLUTION DES MILIEUX RIVERAINS}

L'évolution des milieux riverains est connue grâce aux indices morphosédimentaires et archéologiques situés soit à l'extrémité orientale de la plaine d'Arles (Trébon, site de La Calade), soit sur le fleuve actuel (Avignon et Arles), soit sur les bras fossiles de SaintFerréol et Ulmet dans le delta (Le Carrelet, Cabassole, La Capelière, Mornès) (fig. 4). Globalement, la période considérée semble caractérisée par une activité hydrologique et sédimentaire irrégulière mais modeste, dont l'énergie est comparable à celle du $\mathrm{XX}^{\mathrm{c}} \mathrm{s}$., voire plus faible. Seule la transition Antiquité tardive/Haut Moyen Âge est caractérisée par une crise majeure susceptible de modifier profondément les milieux fluviaux. $\mathrm{Du} \mathrm{V}^{\mathrm{e}} \mathrm{s}$. avant J.-C. au V' $\mathrm{s}$. après J.-C., l'activité hydrosédimentaire n'induit pas de transformations majeures des lits fluviaux. Ses fluctuations sont mieux perçues en milieu deltaïque où la faible dénivellation de la plaine permet une meilleure expression des crues : les débordements y sont 


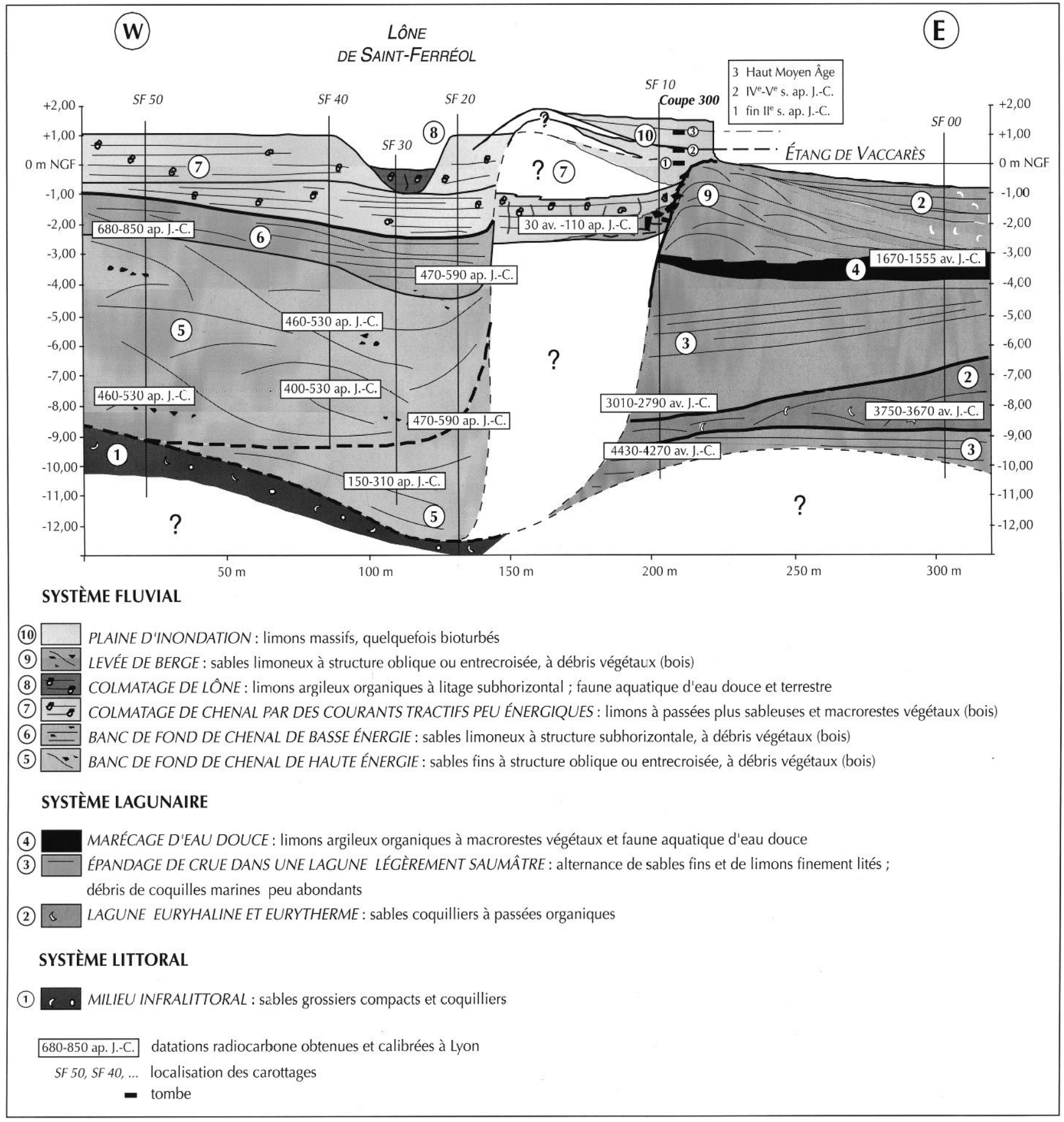

Fig. 8 - Le remblaiement du chenal du fleuve à la fin de l'Antiquité dans le delta du Rhône : site du Carrelet.

plus fréquents et les taux de sédimentation plus significatifs que sur les sites urbains, ancrés sur des affleurements rocheux. On peut néanmoins y distinguer plusieurs phases.
Entre le $V^{t}$ et le II ${ }^{e}$ s. avant J.-C., les crues débordant dans la plaine d'Arles envahissent le Trébon lors de quelques épisodes plus importants (Bruneton, à paraître). À Arles même, leurs dépôts atteignent 

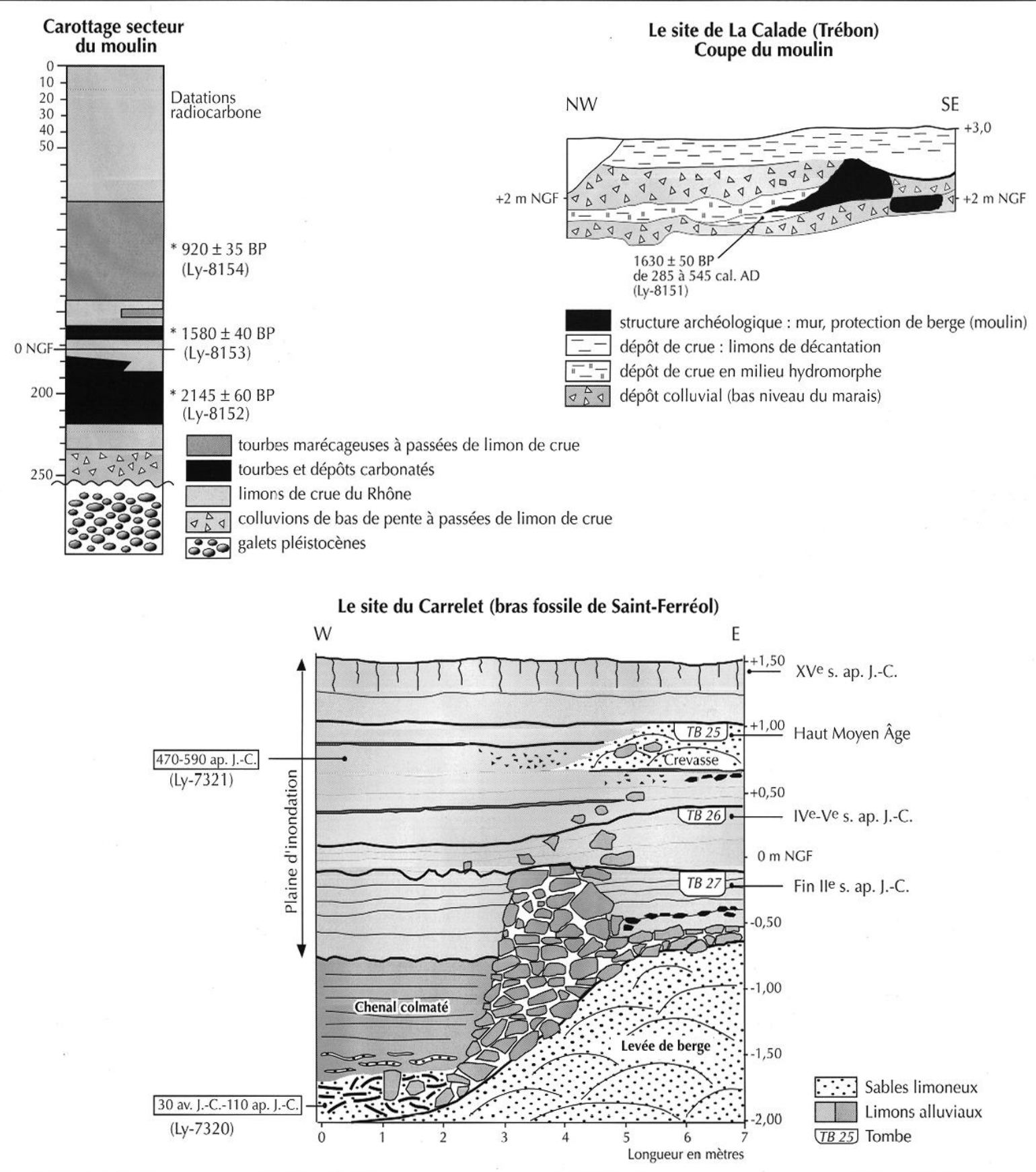

Fig. 9 - Crues, hydromorphie et occupation du sol dans la plaine d'Arles (La Calade) et le delta (Le Carrelet) (d'après Arnaud-Fassetta, 1998).

5,5 m NGF vers 175 avant J.-C. et sont contemporains d'une rétraction durable de l'espace urbain situé dans le champ d'inondation ( $c f$. Arcelin et al., infra, p. 124). Dans le delta, leur compétence est élevée, mais les taux de sédimentation restent moyens à faibles $(0,6-1,2 \mathrm{~mm} / \mathrm{an})$.
À partir de la fin du $\mathbb{I}^{e r}$ s. avant J.-C., l'incision du lit et le bon calibrage du chenal expliquent vraisemblablement l'extension spatiale plus limitée des épisodes de crue, jusqu'à la crise du $\mathrm{V}^{\mathrm{e}} \mathrm{s}$. après J-C. Le fonctionnement fluvial connaît cependant des variations perceptibles (fig. 9) : 
- du Ir s. avant au I ${ }^{\text {er }} \mathbf{s}$. après J.-C., plusieurs crues entretiennent un taux de sédimentation élevé $(2-4 \mathrm{~mm} / \mathrm{an})$ dans le delta, mais leur compétence reste moyenne. Sur les sites urbains d'Arles et Avignon, quelques inondations sont connues au tournant de l'ère : 4 (?) à Avignon vers 16,5 m NGF dans la seconde moitié du I Ir s. avant J.-C ; 2 à Arles vers $3 \mathrm{~m}$ NGF, à la même période. Le développement de marais tourbeux à l'extrémité du lit majeur (Trébon) et l'hydromorphie de la plaine sous le site du cirque d'Arles, au cours du $\mathrm{I}^{\mathrm{er}} \mathrm{s}$. avant J.-C., témoignent de la montée temporaire du système hydrique. Cette tendance n'est cependant pas perçue à Avignon, où un égout du $\mathrm{I}^{\text {er }}$ s. après J.-C. occupe une position relativement basse ;

- dans la seconde moitié du $\mathrm{I}^{\mathrm{er}} \mathrm{s}$. et jusqu'au milieu du $\mathrm{II}^{\mathrm{e}} \mathrm{s}$. après $\mathrm{J}$.-C., les crues ne dépassent pas les berges du fleuve, sauf quelques exceptions dans le delta où leur compétence et leur taux de sédimentation (0,1-1 mm/an) restent néanmoins très faibles. Bien que la berge du Rhône soit peu élevée ( 2 à 3 m NGF) au sud de Beaucaire (site du Mas Neuf), elle porte une voie de circulation et un établissement agricole. À Arles, Avignon et dans le Trébon, aucune trace d'inondation n'a pu être mise en évidence. L'encaissement du chenal induit un bon drainage naturel des sols dans le champ d'inondation : il est pcrccptiblc sous lc cirquc d' $\Lambda$ rles et permet, dans le delta, le développement d'une importante bioturbation ${ }^{6}$ et un début de pédogenèse ;

- de la fin du II ${ }^{\mathrm{e}} \mathrm{s}$. au début du Ve s. après J.-C., les crues deviennent progressivement plus hautes et plus compétentes qu'à la période précédente, mais les taux de sédimentation restent faibles dans le delta $(0,8-1,2 \mathrm{~mm} / \mathrm{an})$. À Arles, un ou deux dépôts de crues recouvrent la piste du cirque vers 2,8 m NGF, ainsi que le quartier de Trinquetaille qui semble plus menacé (Bravard, 1989). La montée du niveau hydrique à Arles à la fin du III $\mathbf{s}$. après J-C. coïncide avec un rehaussement de la voirie et des canalisations.

De la fin $d u V^{t} s$. à la fin du VIres. après J.-C., le fleuve connaît une recrudescence des apports liquides exceptionnels et des apports solides, aboutissant à une métamorphose des milieux fluviaux. Les crues atteignent des cotes très importantes à Avignon (16 m NGF) et Arles

6. Transformation d'un sédiment sous l'action des déplacements d'organismes vivants en son sein. Elle est le plus souvent associée à la pédogenèse.
(5 m NGF), inondant des quartiers habités ou occupés, dont quelques-uns sont alors abandonnés ( $c f$. Arcelin et al., infra, p. 126). Dans le delta, leurs dépôts témoignent de débits plus compétents, avec des taux de sédimentation élevés $(1,3-4 \mathrm{~mm} / \mathrm{an})$. Dans le Trébon, les eaux envahissent épisodiquement l'extrémité orientale du champ d'inondation; la remontée du système phréatique engorge les sols et favorise l'extension de marécages qui entravent le fonctionnement du moulin de La Calade.

Ce renforcement des flux liquides et solides est associé à une métamorphose des milieux alluviaux : le chenal se remblaie rapidement $(6 \mathrm{~m}$ en trois siècles au Carrelet, fig. 8) ; dans le delta, le " tressage " se développe, en particulier sur le bras d'Ulmet, dans les levées de berge duquel se multiplient les lônes ${ }^{7}$ et les brèches. Cette évolution est certainement à l'origine de l'abandon des bras d'Ulmet et de Saint-Ferréol et de l'apparition du bras du Grand Passon, qui fonctionne durant l'époque médiévale.

\section{SYNTHÈSE ET DISCUSSION}

\section{COHÉRENCES ET DISPARITÉS LE LONG DE L'AXE FLUVIAL}

Élaborer une synthèse du fonctionnement rhodanien du lac Léman à la mer au cours de l'Antiquité reste encore un projet hasardeux pour plusieurs raisons. On ne dispose pas en effet d'informations comparables tout le long de l'axe fluvial : plus riches sur les sites urbains, qui ont bénéficié de fouilles systématiques, elles sont plus dispersées et plus disparates en milieu rural. Sur le Rhône moyen, les recherches ont, en outre, plutôt porté sur les affluents et la plaine alluviale distale que sur les milieux fluviaux rhodaniens stricto sensu. Les effets de sites peuvent « brouiller les cartes » : les hauteurs, la turbulence et l'expansion des eaux de crues ne sont pas les mêmes selon la largeur et la topographie des lits. Vienne, encaissée entre des versants élevés, pourra ainsi apparaître plus menacée que Lyon ou Arles qui disposent de plus vastes espaces où se déployer.

Le fleuve lui-même ne possède pas la même énergie dans tous les secteurs, selon sa pente longitudinale et les

7. Bras mort ou faiblement actif du Rhône, momentanément réactivé lors des crues. 


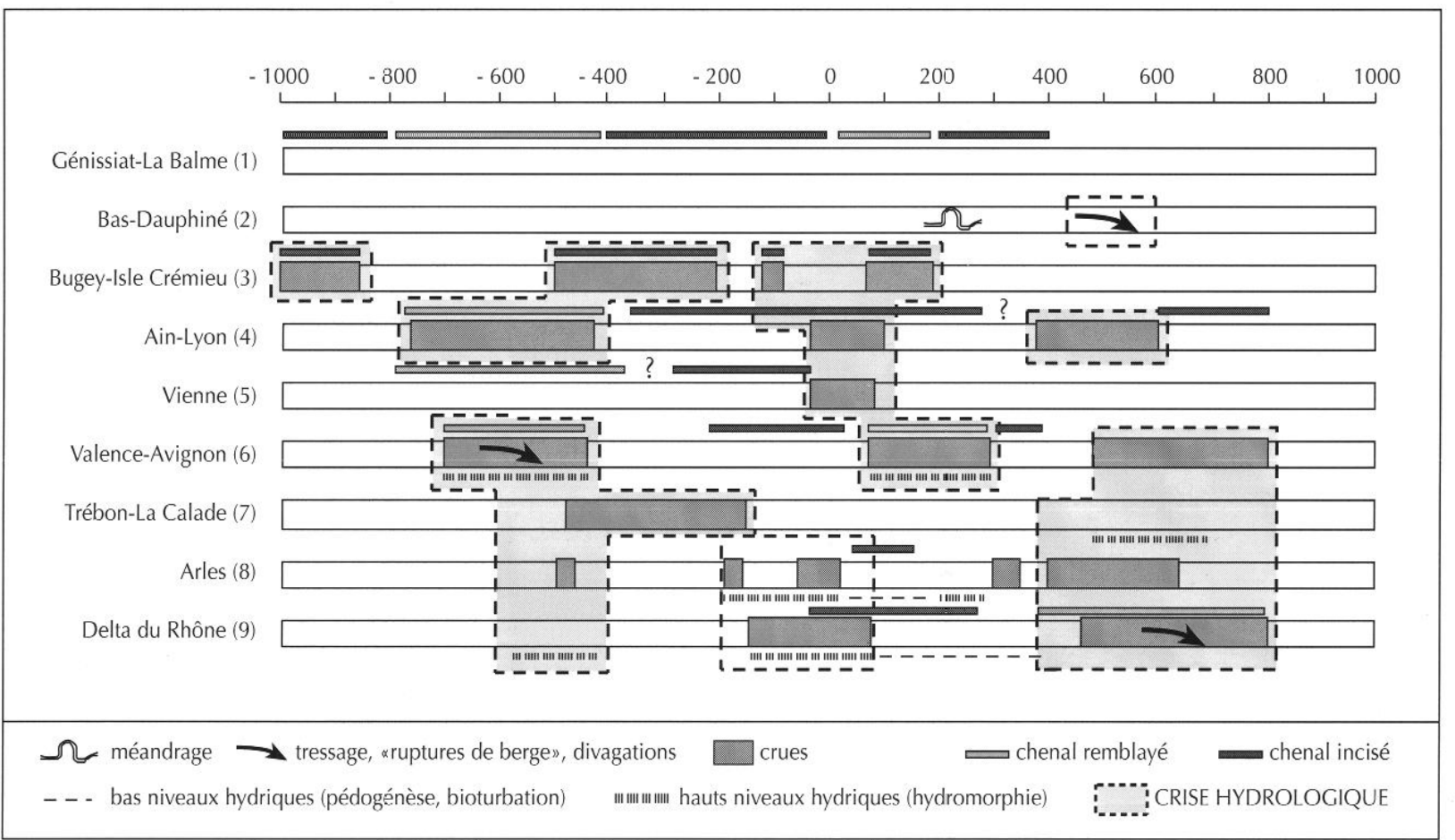

Fig. 10 - Évolution de la dynamique alluviale du Rhône, du Léman à la mer : 1, Savay-Guerraz (1985), Magny et Richard (1985), Bravard (1987) ; 2, Roberts et al. (1997) ; 3, Salvador (1991) ; 4, Bravard et al. (1992) ; 5, Bravard et al. (1990) ; 6, Berger (1996a et b) ; 7, Bruneton (à paraître); 8, Arcelin et al. (cf. infra, p. 121-129); 9, Arnaud-Fassetta (1998).

apports solides et liquides de ses affluents. Certains bassins, comme celui d'Orange, peuvent ainsi favoriser l'accrétion ${ }^{8}$ fluviale et la persistance d'îles. Le Tricastin et la Valdaine sont soumis à un contexte hydrologique particulier, lié aux charges solides considérables des affluents de rive gauche, entre la confluence de la Drôme et celle de l'Ouvèze.

Enfin, le transfert progressif de la charge de fond vers l'aval peut induire des décalages dans les changements d'état des lits, plus précoces en amont.

La cohérence des données acquises doit cependant être soulignée, même s'il subsiste quelques disparités (fig. 10).

Les deux crises majeures, au cours du Premier Âge du Fer et à partir du $I^{\boldsymbol{e}}$ s. et surtout du $V^{\boldsymbol{t}}$ s. après J.-C., sont perçues sur tous les secteurs, à l'exception du Rhône inférieur où les indices de la première sont masqués par l'épaisseur de l'accumulation. La seconde correspond à une augmentation des débits liquides extrêmes (avec un régime

8. Accroissement progressif d'un dépôt par sédimentation. contrasté ?) et de la charge solide des rivières. Elle est donc caractérisée par la fréquence de crues puissantes et chargées. Elle suppose un déstockage détritique important, confirmé par l'analyse minéralogique des sédiments du delta : l'ensemble des bassins-versants, y compris la basse Durance, contribue alors à l'alimentation alluviale. Ces désordres induisent une métamorphose des lits fluviaux qui a certainement modifié les conditions d'habitat et d'exploitation des milieux riverains.

On peut penser que la mise en culture des hauteurs, amorcée à partir du IV ${ }^{*}$ s. après J.-C., a contribué à augmenter l'érosion dans les bassins-versants, donc les débits solides des cours d'eau, et amplifié les phénomènes de crue (Berger, 1995). Des épisodes d'incendies récurrents, avec un pic autour du $\mathrm{VI}^{\mathrm{c}} \mathrm{s}$. après J.-C., sont observés en moyenne vallée du Rhône sur les versants et les plateaux de l'avant-pays rhodanien et dans les basses plaines fluviales.

Entre le $V^{k} s$. avant et le $I V^{t}-V^{t} s$ s. après J.-C., c'est-à-dire en fait pendant toute l'Antiquité romaine, les niveaux et la fréquence des débordements sont plus faibles, la plaine 
d'inondation mieux drainée, en relation avec un chenal bien incisé. Les données sont imprécises entre le $\mathrm{V}^{c}$ et le $\mathrm{II}^{\mathrm{e}}$ s. avant $\mathrm{J}$.-C., qui correspond vraisemblablement à une période de transition encore marquée par une certaine instabilité du fleuve. Mais, à partir du début du $\mathrm{II}^{\mathrm{c}} \mathrm{s}$. avant et jusqu'au $V^{c} s$. après J.-C., soit sur plus de six siècles, les inondations recensées sur les sites urbains sont peu nombreuses ( 7 ou 8 , ce chiffre étant sans doute sous-évalué). Le chenal y est donc calibré pour évacuer les crues de période de retour cinquantennale à centennale. Cette période correspond à un régime fluvial plus régulier, avec une alimentation détritique réduite, ce qui n'exclut pas la récurrence de quelques crues importantes.

Des disparités subsistent, en particulier dans la moyenne vallée du Rhône qui apparaît hétérogène dans ce schéma global entre le I ${ }^{\mathrm{er}} \mathrm{s}$. avant et la fin du $\mathrm{III}^{\mathrm{c}} \mathrm{s}$. après J.-C.

Elle n'enregistre pas, en effet, la recrudescence des crues débordantes perçue au cours des dernières décennies du I Ir s. avant J.-C. sur le Rhône supérieur et jusqu'au milieu du I ${ }^{\mathrm{er}} \mathrm{s}$. après J.-C. sur le Rhône inférieur, associée à une hydromorphie des sols alluviaux. Ces crues n'atteignent cependant jamais le niveau et l'énergie des crues de l'Antiquité tardive, ni des événements de 1993-1994 (Arnaud-Fassetta, 1998). On soulignera que le Rhône inférieur est alors allogène dans le midi méditerranéen, qui est caractérisé par une remarquable stabilité de son géosystème (Provansal, 1996) : les minéraux lourds montrent que les apports solides du fleuve dans le delta proviennent en fait du bassin amont du Rhône ou du Massif central. Les crues et la montée des lits fluviaux enregistrées à la même époque sur le Vidourle, en Languedoc, pourraient confirmer cette dernière hypothèse.

Entre la fin du I ${ }^{\mathrm{er}}$ s. et la fin du III $^{\mathrm{c}}$ s. après J.-C., la Valdaine et le Tricastin enregistrent en revanche des taux de sédimentation élevés et une augmentation de l'hydromorphie. Ces caractères sont apparemment en contradiction avec l'absence de manifestations hydrologiques excessives en Provence à la même époque et avec le fonctionnement modéré du fleuve lui-même, tant dans son cours amont qu'en aval d'Orange.

La remontée du niveau des aquifères pourrait être liée à la diminution des processus d'évaporation et d'évapotranspiration, qui expliquent la réapparition durable du Hêtre, du Sapin et du Pin sylvestre (Thiébault et Rousseau, com. pers.) ; ce phénomène pourrait être associé à l'exhaussement des lits des affluents de rive gauche du Rhône (Berger, 1996b), créant un effet de barrage latéral pour le drainage naturel de la plaine. Cette crise torrentielle des affluents préalpins soulève ellemême la question d'une détérioration climatique fraîche et humide. Celle-ci est d'ailleurs enregistrée par les lacs circum-alpins (Magny, 1992), par les glaciers suisses et autrichiens et, plus généralement, par les paléoenvironnements de l'Europe du Nord-Ouest qui témoignent d'un regain d'humidité au cours des deux premiers siècles de notre ère. Il est possible que cette pulsion climatique soit plus fortement enregistrée par les petits organismes préalpins que par le vaste bassin rhodanien. On peut émettre aussi l'hypothèse d'une géographie climatique qui limiterait à la moyenne vallée du Rhône la pénétration des influences maritimes océaniques durant les deux premiers siècles de notre ère, situant ainsi vers Orange la limite nord du climat méditerranéen.

\section{LES HOMMES ET LE FLEUVE}

Le Rhône est un grand axe de communication, dont on connaît l'importance par l'activité des ports fluviaux de Lyon, Vienne, Avignon et Arles au cours de l'Antiquité ( $c$. Christol et Fiches, infra, p. 111-155). Dans le delta, les sites de berges sont abandonnés à la fin du premier millénaire, à la suite des défluviations qui donnent naissance au bras médiéval : leur utilisation du fleuve en devient $a$ contrario évidente.

La navigation est évidemment plus facile quand le chenal est bien incisé... mais, étant donné le faible tirant d'eau des navires, le fleuve n'est-il pas toujours navigable par des bateaux à fond plat, quelle que soit la période considérée ? Le problème est peut-être plus important en aval d'Arles ou de Beaucaire qui correspondent au point de rupture de charge des bateaux de mer : le trajet est-il sans difficultés pour ces bâtiments à quille, dans un lit encombré de sédiments comme c'était le cas au Ve-VIII' s. ?

La périodisation des dynamiques fluviales peut être partiellement synchronisée avec les contraintes qui conditionnent l'occupation du sol, mais sans systématisme.

Les crues ne jouent pas un rôle déterminant sur la localisation et la durée des sites d'habitat : leur caractère épisodique ne perturbe pas durablement les activités. L'élévation de leur niveau et leur plus grande fréquence 
restreignent-elles l'expansion de l'espace urbain (Arles au II ${ }^{\mathrm{e}}$ s. avant J.-C., Vienne et Lyon au début du $\mathrm{I}^{\mathrm{er}}$ s. après J.-C.) ? ou obligent-elles seulement à des travaux de protection de berges (Lyon, Arles et le site du Carrelet au $\mathrm{I}^{\mathrm{er}}$ s. après J.-C.) ? La contrainte fluviale peut cependant jouer pour les petits habitats dispersés et les structures viaires implantées dans les zones les plus sensibles hydrologiquement, en milieu rural. Ainsi à la confluence Rhône/Aygues, dans la plaine d'Orange, quelques sites et installations agricoles et une voie empierrée importante et large, implantée dans l'axe d'un decumanus du cadastre $\mathrm{B}$ d'Orange, ont une durée d'existence très brève. Ils sont enfouis sous les dépôts alluviaux épais déposés entre le I ${ }^{\text {er }}$ et le III $^{\text {e-IV }}{ }^{\mathrm{e}}$ s. Des sols hydromorphes et un couvert forestier ouvert (d'après les analyses malacologiques) prouvent un abandon temporaire des lieux. Des observations identiques ont été effectuées dans le sud du Tricastin, dans la zone de Mondragon-Les Ribauds, où un exhaussement important du plancher alluvial est perceptible entre le début de notre ère et l'Antiquité tardive.

Le drainage naturel des sols associé à un encaissement probable des lits fluviaux permet une exploitation agricole intensive de la plaine alluviale. Sur le Rhône moyen, au cours de la dernière moitié du ${ }^{\mathrm{er}} \mathrm{s}$. avant J.-C. ou de la première moitié du I ${ }^{\mathrm{er}}$ s. après J.-C., cette exploitation ne nécessite pas de drainage artificiel : ainsi sur le site des Girardes à Lapalud (Vaucluse), le réseau de fossés drainants, juste antérieur ou contemporain de la fin $\mathrm{du} \mathrm{I}^{\mathrm{cr}} \mathrm{s}$. après J.-C., recoupe des trous de plantation reliés à la culture de la vigne et datables du ${ }^{\text {er }}$ s. avant J.-C. (datation radiocarbone en cours) ; la formation d'horizons calciques au cours de la même période démontre un déficit hydrique ou une phase d'évapotranspiration importante (confirmée par les études palynologiques). Dans le Bas-Rhône, en revanche, l'expansion agricole romaine ne semble possible qu'associée à un drainage artificiel, rendu possible par un contexte d'abaissement naturel du niveau phréatique et de l'écoulement fluvial.

La montée du niveau hydrique, associée à une métamorphose des milieux fluviaux, est bien plus contraignante. Dans les villes, elle coïncide avec des réaménagements (remblais, exhaussements des réseaux d'évacuation des eaux et, parfois, des réseaux viaires et des structures bâties), dont on ne sait s'ils sont liés à des choix urbanistiques ou à la nécessité de s'abriter des inondations.
C'est sans doute dans l'espace agricole qu'elle induit le plus de changements... mais ils sont plus difficiles à percevoir par l'archéologie. La contrainte du fleuve doit non seulement y être perçue par rapport à l'habitat et sa sensibilité, mais encore par rapport aux sols qui conditionnent les possibilités et les modes d'exploitation. Au cours des épisodes de crise hydrosédimentaire, la montée du système hydrique a nécessité l'élaboration d'importants réseaux de drainage afin de rabattre le niveau des nappes. C'est le cas pour la plus grande partie de la période allant du milieu ou de la fin du $\mathrm{I}^{\mathrm{cr}} \mathrm{s}$. au $\mathrm{III}^{\mathrm{e}} \mathrm{s}$. après J.-C. en moyenne vallée du Rhône et du $\mathrm{V}^{\mathrm{c}}-\mathrm{VI}^{\mathrm{c}} \mathrm{s}$. au $\mathrm{VII}^{\mathrm{e}}$-VIII ${ }^{\mathrm{e}}$ s. après J.-C. dans l'ensemble des régions considérées.

Le Haut Moyen Âge, avec l'abandon de l'entretien des réseaux de drainage, semble correspondre à une paludification de l'ensemble des lits majeurs. La remontée durable des aquifères est confirmée par la nature des paléosols, la malacofaune et les microfossiles non polliniques dans le bassin valdainais et la plaine d'Orange : les paysages végétaux identifiés révèlent une extension de prairies humides et de zones palustres, dévolues à l'exploitation pastorale. Les ripisylves et la chênaie caducifoliée tiennent une place importante. Dans le Trébon, l'engorgement et l'atterrissement des drains et des chenaux secondaires obligent à abandonner le Moulin de La Calade (Bruneton, à paraître).

$$
\text { * }
$$

Malgré quelques disparités dans l'évolution de ses milieux fluviaux, dont on a montré qu'elles tenaient souvent aux conditions topographiques ou structurales locales, les fluctuations hydrologiques du fleuve paraissent assez homogènes du Jura à la mer. Le risque que le fleuve fait peser sur ses rives et les modifications qu'il impose à l'environnement sont pratiquement contemporains au cours des deux millénaires étudiés, du lac Léman à la mer. Cette homogénéité souligne l'importance du facteur climatique à l'échelle du bassinversant.

Les recherches ont mis en évidence des fluctuations d'ordre pluridécennal à séculaire : après la phase d'intense activité fluviale du Premier Âge du Fer, la période qui s'étend du Second Âge du Fer au Ve s. après J.-C. est globalement favorable à l'installation des sites urbains à proximité immédiate du chenal et à l'extension de l'agriculture dans la basse plaine. Seul le Rhône moyen pré- 
sente une exception momentanée, du $\mathrm{I}^{\mathrm{er}}$ au III ${ }^{\mathrm{e}}$ s., dont il est discuté dans le texte. La détérioration des conditions d'occupation de la vallée à partir du $\mathrm{V}^{\mathrm{e}} \mathrm{s}$. est manifeste.

\section{Remerciements}

Nous remercions ici les services municipaux d'Archéologie des villes de Lyon, Avignon et Arles, l'AFAN, les services régionaux de l'Archéologie (Rhône-Alpes et PACA), l'ensemble des responsables d'opération du secteur II du TGV-Méditerranée Valence/Avignon, C. Jung (programme fossés), F. Magnin (malacologie), T. Odiot (prog. H11 et TGV) et V. Bel (TGV), J.-L. Brochier et A. Beeching (ATP du CAPV). Merci à Ph. Leveau qui nous a donné l'occasion de bâtir cette synthèse et nous a fait bénéficier d'une indispensable critique. 